\title{
Effect of Air Swirler Profile on Spray Characteristics
}

\author{
Shraddha Sharma, Srikrishna Sahu, Thirumalachari Sundararajan \\ ${ }^{1}$ Indian Institute of Technology, Madras \\ Chennai, TamilNadu, India \\ me14d411@smail.iitm.ac.in
}

\begin{abstract}
Pressure swirl atomizers are widely used in gas turbines and liquid propellant rockets to obtain improved atomization. In these atomizers, the liquid to be atomized enters through tangential inlets producing a swirling motion inside a swirl chamber and creates a central gas core surrounded by a liquid sheet that spreads radially outward from the orifice exit. In a typical gas turbine combustor coaxially fitted swirler-atomizer assembly is used for better performance. In this paper, an experimental study has been conducted to investigate the effect of the swirler profile on a co-axial hollow cone spray. A coaxially fitted air swirler and atomizer assembly is used for the analysis. High speed imaging is used to capture the sharp images of spray and for the calculation of spray cone angle. Phase Doppler Interferometer (PDI) is used to analyze SMD variations. Significant change in spray characteristics is observed with different air flow rates and swirler shapes.
\end{abstract}

Keywords: Air swirler, Flat vane, Curved vane, Spray.

\section{Introduction}

The design of an efficient combustor requires several considerations, especially to meet the stringent regulation regarding emission levels in the exhaust. These requirements include stability limits, high combustion efficiency, high intensity of heat release, low burner pressure drop and low emissions. Gas turbine combustors are continuous combustion type internal combustion engine which requires long sustainability of flame. One of the most effective ways of flame stabilization is induction of swirling flow in the primary zone of combustor by the mounting of an air swirler in the dome of the combustor. The intensity of swirl flow is characterized by Swirl Number. Geometry based swirl number $\left(\mathrm{S}_{\mathrm{G}}\right)$ is defined as:

$$
S_{G}=\frac{2}{3}\left\{\frac{1-\left(\frac{D_{h}}{D_{t}}\right)^{3}}{1-\left(\frac{D_{h}}{D_{t}}\right)^{2}}\right\} \times \tan \alpha
$$

where $D_{h}$ and $D_{t}$ are hub and tip diameters of the swirler and $\alpha$ is vane angle.

Strong swirling flow develops a low pressure region downstream swirler exit which is known as recirculation zone. This recirculation zone anchors the flame by continuously mixing the unburnt fuel- air mixture with hot combustible products. Air swirler generated recirculation zone provides better mixing than is normally obtained by other means, such as bluff bodies, because swirl components produce strong shear regions, high turbulence, and rapid mixing rates [1]. The aerodynamics of swirling flow influences the combustion performance significantly as it directly affects the fuel-air mixing and the flow residence time [2]. Kilik [3] experimentally determined, the influence of swirler geometric parameters such as vane angle, space-to-chord ratio and aspect ratio, on the size and turbulence characteristics of the downstream recirculation region. It was concluded that curved vanes operate much more efficiently than flat vanes due to the fact that the flat vanes always run under 'stalled' conditions; due to which larger wakes are created behind flat vanes and greater momentum loss occurs. Since the momentum loss exhibits itself in the form of a pressure drop, therefore, conventional flat vane swirlers give rise to much higher pressure losses than curved vane swirlers do. Khandelwal et al. [4] computationally analysed the effect 
of geometric parameters of swirler (vane numbers and vane angle) and inlet mass flow through swirler on the flow characteristics. It was found that the axial reverse flow velocity, turbulence along axial direction and pressure drop increase with increase in vane angle but at the cost of pressure drop. Increase in mass flow rate was found to decrease the pressure drop through swirler and constant pressure drop was observed when mass flow rate was increased beyond a critical value.

Pressure swirl atomizer is widely used in gas turbine combustor for fuel injection due to its ease in manufacture. Pressure swirl atomizer rely on the conversion of pressure energy of the bulk liquid into kinetic energy of dispersed droplets. The liquid is fed through tangential ports into a swirl chamber mounted upstream of the discharge orifice. A thin conical liquid sheet is formed at the discharge orifice which spreads at a certain angle due to centrifugal forces. Disturbances propagating on the surface of this sheet cause its disruption into ligaments and finally into droplets in the form of a hollow cone spray [5]. An efficient combustion process requires the presence of fine and uniformly distributed droplets in the combustor. Modern combustors use a coaxially fitted air swirler and atomizer assembly i.e. Lean direct injection (LDI) strategy, to achieve enhanced spray breakup. In LDI, high degree of swirling airflow surrounding a fuel injector facilitates the atomization of the fuel streams into very fine drops. Since LDI features direct injection of fuel and air into the combustion chamber thus, the performance of LDI heavily depends on the configuration of air swirler[6]. Rosa et al. [7] studied the effects of swirl on the performance of a liquid spray for flat vane axial swirlers and concluded that flow reversal of the drops occurs at a high swirl number within the recirculation region. Also, the spatial dispersal of drops increases with increase in swirl strength while the concentration of large drops increases near the core of the swirling field with increasing swirl number. Sanadi et al. [8] experimentally investigated the spray characteristics of a hollow-cone spray in an unconfined, low speed co-annular isothermal swirling air jet environment. A guided vane swirler with a vane angle of $45^{\circ}$ (corresponding geometric swirl number $\mathrm{SG}=0.8$ ) was used to impart swirling motion to the co-axial airflow. Liquid flow rate was fixed whereas various levels of co-flowing annular swirling air were imparted. The results indicated that Sauter mean diameter (SMD) in the radial direction is highly dependent on the behaviour of swirling air imparted to the spray and the hollow cone tends to entrap the swirl stream and swirling air causes fanning out of the spray droplets.

In this work, a flow geometry similar to lean direct injection (LDI) combustors is considered, i.e. injection of a hollow cone spray from a pressure swirl atomizer into a swirling air flow field. The intent of this work is to provide qualitative insight into the nature of atomization performance with varying swirler shape and swirl air flow rate. The variation in spray cone angle is measured by the Shadowgraph technique. Phase Doppler Interferometer (PDI) technique is used to measure droplet behaviour.

\section{Methodology}

A schematic diagram of the experimental set-up consisting of coaxially fitted air swirler and simplex atomizer is shown in Fig. 1. The schematic of flat and curved air swirlers are shown in Fig. 2. Air and water are used as working fluids at ambient temperature. The dimensional details of swirlers are presented in Table 1.

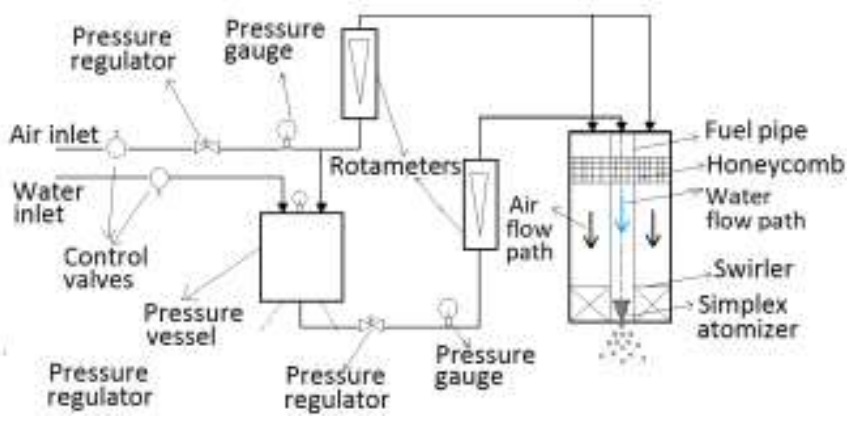

Fig. 1: Schematic of test section. 


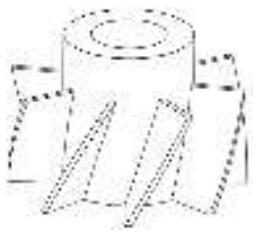

(a) flat vane swirler.

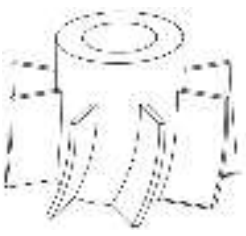

(b) curved vane swirler.

Fig. 2: Schematic of air swirlers.

Table 1: Swirler dimensions.

\begin{tabular}{|c|c|c|c|c|c|}
\hline S.No. & Swirler type & Vane angle $(\alpha)$ & $\begin{array}{l}\text { Hub diameter }\left(\mathrm{D}_{\mathrm{h}}\right) \\
(\mathrm{mm})\end{array}$ & $\begin{array}{l}\text { Tip diameter }\left(\mathrm{D}_{\mathrm{t}}\right) \\
(\mathrm{mm})\end{array}$ & No. of vanes \\
\hline 1. & Flat & $30^{\circ}$ & 41 & 75 & 8 \\
\hline 2. & Curved & $30^{\circ}$ & 41 & 75 & 8 \\
\hline
\end{tabular}

Simplex atomizer consists of orifice diameter of $1 \mathrm{~mm}$ and three tangential ports of $0.8 \mathrm{~mm}$ diameter at $120^{\circ}$ apart. The orifice diameter of the atomizer is $1 \mathrm{~mm}$.

PCO Dimax high speed camera is used to capture 2000 instantaneous images of spray at $10000 \mathrm{fps}$. These images are further processed in ImageJ software to for spray cone angle calculation. Artium Phase Doppler interferometer (PDI) 300 is used for the analysis of the radial distribution of Sauter mean diameter (SMD). In the basic PDI optical system, the laser beam is split into two beams of equal intensity. The beams are then focused and made to intersect using a transmitter lens. Frequency shifting is used to compress the frequency dynamic range and to resolve the direction ambiguity that would occur for drops passing in a reverse direction. Particles passing through the beam intersection scatter light that is collected by the receiver lens. The phase difference between the signals provides droplet diameter and frequency difference provides velocity of the droplets. The injection pressure and flow rate of water are maintained at 3 bar and $0.939 \mathrm{lpm}$, throughout the experiment. The air flow rate is varied from 0 to $2000 \mathrm{lpm}$.

\section{Results}

\section{1. Spray cone angle variation}

Spray cone angle governs the dispersion of spray and it is defined as an initial angle of conical sheet formed at the nozzle exit. The images of spray captured by high speed camera at 3 bar liquid injection pressure and 2000 lpm air flow rate are shown in Fig. 3 (a) and (b).

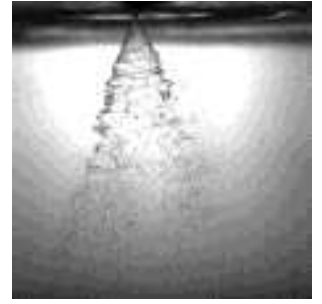

(a) $30^{\circ}$ flat vane

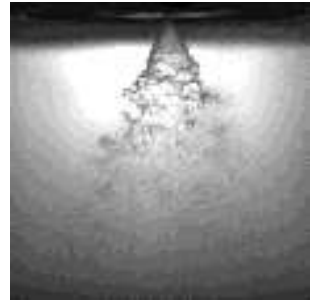

(b) $30^{\circ}$ curved vane

Fig. 3: Instantaneous images of spray.

The variation of spray cone angle with swirl air flow rate is shown in Fig. 5. Spray cone is calculated at 40mm swirler exit. It is observed that the spray cone angle increases with air flow rate and a wider dispersion are obtained for curved vane swirler as compared to a max of the flat vane. The maximum spray cone angle obtained with flat vane swirler is $62.99^{\circ}$ 
whereas for the curved vane swirler it is $64.57^{\circ}$. The use of curved vanes provides smooth guidance to the flow and prevents the flow separation taking place in case a flat vane swirler which results in greater centrifugal pressure and therefore, enhancement in spray cone angle is obtained.

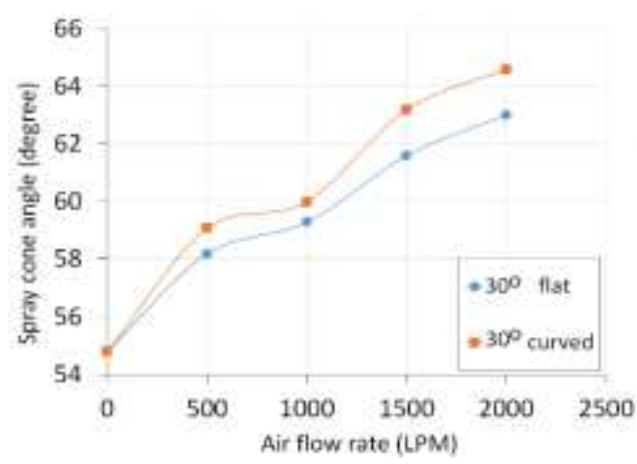

Fig. 4: Spray cone angle variation.

\section{2. Sauter mean diameter variation}

Sauter mean diameter (SMD or $\mathrm{D}_{32}$ ) is the diameter of a hypothetical droplet whose ratio of volume to surface area is equal to that of the entire spray. It is represented as:

$$
S M D=\left(\frac{\sum_{i=1}^{N} n_{i} d_{i}^{3}}{\sum_{i=1}^{N} n_{i} d_{i}^{2}}\right)
$$

Where,

SMD: Sauter mean diameter

$\mathrm{n}_{\mathrm{i}}=$ Number of droplets in size range $\mathrm{i}$

$\mathrm{d}_{\mathrm{i}}=$ Average diameter of droplets in size range $\mathrm{i}$.

Sauter mean diameter is a good way to describe a spray that is used for processes involving evaporation because it deals with a surface area. To enhance droplet evaporation one has to maximize the active surface area and minimize the internal volume of the droplet. Thus, the lower the SMD, the more rapid is the evaporation process. Measuring drop size distributions at different locations and making internal comparisons, provide a better understanding of spray characteristics.

Figure 5 represents the radial variation of Sauter mean diameter (SMD) of spray droplets at 40mm downstream of the orifice exit at $2000 \mathrm{lpm}$ air flow rate and 3 bar liquid injection pressure. As the air flow increases the SMD value decreases because the higher velocity of air causes higher shear at liquid air interface and results in faster atomization. Curved vane swirler results in smaller SMD values as compared to the flat vane swirler. This can be attributed to the absence of the flow separation when curved vane swirler is used due to which the shear imparted on the liquid air interface is more for a curved vane swirler as compared to the flat vane swirler. 


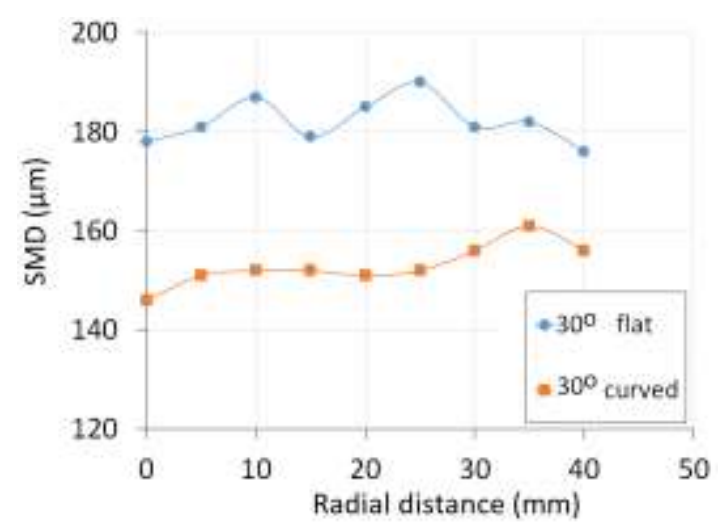

Fig. 5: Sauter mean diameter (SMD) variation.

\section{Conclusion}

Effects of swirler profile (flat and curved) on a hollow cone coaxial spray has been experimentally investigated in this study. Results show that even a slight modification in swirler design influences the spray behaviour a lot. Increase air flow results in higher spray cone angle and higher spray cone angle is obtained for swirler with curved vane profile. Significant reduction sauter mean diameter of spray is observed when curved profile is used. Moreover, curved vane swirler seems to result in uniform distribution of droplets also. Thus, the use of a curved vane swirler in gas turbine combustor is expected to increase the quality of atomization and flame stability.

\section{Acknowledgements}

Authors are thankful to National Centre for Combustion Research and Development (NCCRD), IIT Madras for providing high speed camera and Phase Doppler Interferometry (PDI) to perform the experiments.

\section{References}

[1] N. Syred and J. M. Beer, "Combustion in swirling flows: a review," Combustion and flames, vol. 23, no. 2, pp. 143201, 1961.

[2] J. F. Driscoll, R. H. Chen, and V. Tangirala, "The role of recirculation in improving internal mixing and stability of flames," in 25th AIAA Aerospace Sciences Meeting, 1987, pp. 306.

[3] E. Kilik, "The influence of swirler design parameters on the aerodynamics of downstream recirculation region," Ph.D. dissertation, Cranfield school of technology, UK.

[4] B. Khandelwal, D. Lili, and V. Sethi, "Design and study on performance of axial swirler for annular combustor by changing different design parameters," Journal of the Energy Institute, vol. 87, no. 4, pp. 372-382, 2014.

[5] A. Lefebvre, Atomization and Sprays. CRC Press, 1988.

[6] S. Archer and A. K. Gupta, "Effect of swirl and combustion on flow dynamics in lean direct injection gas turbine Combustion," in Proceedings of 41st AIAA Aerospace Sciences Meeting and Exhibit, 2003, p. 1343.

[7] A. B. de La Rosa, G. Wang, and W. D. Bachalo, "The effect of swirl on the velocity and turbulence fields of a liquid spray," Journal of Engineering for Gas Turbines and Power, vol. 114, no. 1, pp. 72-81, 1992.

[8] D. Sanadi, K. Rajamanickam, and S. Basu, "Analysis of hollow-cone spray injected in an unconfined, isothermal, coannular swirling jet environment," Atomization and Sprays, vol. 27, no. 1, 2017. 